\title{
Quantum effects in magnetotransport of InGaAs quantum wells with remote Mn impurities
}

\author{
Leonid Oveshnikov ${ }^{1,2, \star}$, Leonid Morgun ${ }^{2,3,4}$, Elena Nekhaeva ${ }^{1}$, Vladimir Kulbachinskii ${ }^{1,5}$, and Boris Aronzon ${ }^{1,2}$ \\ ${ }^{1}$ National Research Center "Kurchatov Institute", 123182 Moscow, Russia \\ ${ }^{2}$ P.N. Lebedev Physical Institute RAS, 119991 Moscow, Russia \\ ${ }^{3}$ Moscow Institute of Physics and Technology, 141700 Dolgoprudnyi, Moscow Region, Russia \\ ${ }^{4}$ National Research University Higher School of Economics, 101000 Moscow, Russia \\ ${ }^{5}$ M. V. Lomonosov Moscow State University, 119991 Moscow, Russia
}

\begin{abstract}
We have studied magnetoresistance and Hall effect of GaAs/ $/ \ln _{x} \mathrm{Ga}_{1-x}$ As quantum wells with remote Mn impurity. Temperature and magnetic field dependencies of samples resistivity indicate several effects related to the magnetic subsystem. Shubnikov - de Haas oscillations indicate the presence of several types of regions in conduction channel with significantly different hole mobilities. We discussed the impact of magnetic impurities on quantum corrections to conductivity by comparing our results with the data for similar non-magnetic structures. Our results suggest that the presence of $\mathrm{Mn}$ atoms leads to the damping of quantum corrections in in the investigated structures.
\end{abstract}

\section{Introduction}

Two-dimensional (2D) semiconductor structures with magnetic impurities are considered to be one of the most promising as a basic system of next-generation spintronics [1]. Substantial part of related publications are focused primarily on unique effects driven by magnetic subsystem (such as anomalous Hall effect (AHE)) [1-4], while the impact of magnetic impurities on more conventional properties of different systems, such as "metal-insulator" transition and quantum oscillations, is much less discussed in literature.

Introducing magnetic impurities into semiconductor matrix at concentrations high enough for the related effects (e.g. ferromagnetism) to be observed usually turns systems into strongly disordered media, for which quantum corrections should be significant. Conventional theory predicts that ferromagnetic phase tends to destroy quantum interference correction, while experimental data for such systems can be rather ambiguous [5-7]. Moreover, electron-electron $(e-e)$ interaction in the presence of ferromagnetic subsystem is relatively poorly studied.

Here we have studied magnetotransport properties of $\mathrm{GaAs} / \mathrm{In}_{x} \mathrm{Ga}_{1-x}$ As quantum wells with separated by spacer $\mathrm{Mn}$ impurity. Spatial separation of acceptor Mn atoms and conducting holes leads to high mobility values of the latter along with feasibility of magnetic interactions between $\mathrm{Mn}$ ions. One of the reasons for interest to these studies is the recent experimental investigation of analogous systems with hopping conductivity, for which the indica-

\footnotetext{
^e-mail: Oveshln@gmail.com
}

tions of "topological" Hall effect contribution have been observed [2].

As far as we know, quantum effects in magnetotransport of systems with spatially separated conducting and magnetic subsystems are poorly studied.

\section{Samples}

The investigated structures were synthesized by MOCVD method on semi-insulating GaAs substrates (schematic structure of the similar samples is published in Ref's [24]). Quantum well (QW) $\operatorname{In}_{x} \mathrm{Ga}_{1-x}$ As with $x \approx 0.2$ was sandwiched between carbon (lower) and manganese (upper) impurity layers separated by GaAs spacers. Samples with different Mn content in the range 0.4 to 0.5 monolayers (ML) were mesa etched into standard Hall bars. Temperature and magnetic field dependencies of transverse $R_{x x}$ and Hall $R_{x y}$ were measured in the temperature interval $2 \mathrm{~K}$ $<T<77 \mathrm{~K}$ and in magnetic field $B$ up to 9T. Additionally, the magnetoresistance of $0.5 \mathrm{ML}$ sample was studied for $B$ $= \pm 1 \mathrm{~T}$ at temperatures down to $0.3 \mathrm{~K}$.

Previous studies of analogous samples showed that $\mathrm{Mn}$ atoms strongly diffuse into nearby layers forming comparatively thick (3-5 nm) $(\mathrm{Ga}, \mathrm{Mn})$ As layer [8]. Mn atoms in GaAs crystal matrix form electrically charged substitutional and interstitial defects. Inhomogeneous distribution of $\mathrm{Mn}$ in the impurity layer gives rise to the fluctuation potential (FP). FP forms additional potential wells in the conduction channel that leads to the "metal-insulator" transition for structures with sufficiently low Fermi energies. Theory predicts that in insulating state conduction channel in such structure consists of metallic droplets in- 


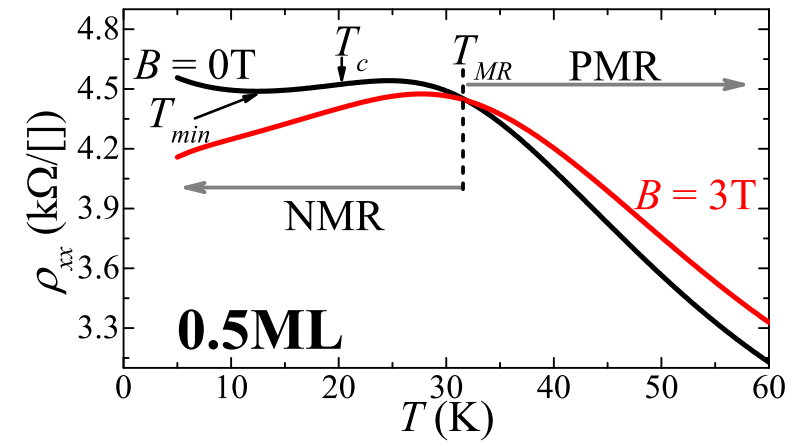

Figure 1. (colour online) Temperature dependencies of resistivity for $0.5 \mathrm{ML}$ sample at $B=0$ and $3 \mathrm{~T}$. Temperature ranges of positive and negative MR are marked, along with several characteristic temperatures (see text).

serted into insulating matrix [9]. Significant difference of Hall mobility and estimated from Shubnikov - de Haas (SdH) oscillations mobility was experimentally observed for these structures [10]. Moreover, SdH oscillations were observed even for samples with hopping conductivity. It suggests the presence of at least two types of consecutively connected areas in QW with different mobilities, which is in good agreement with theory. It is important to note that mobility difference for these two types of areas remains significant even for samples with drift conductivity (including samples studied here).

\section{Results and discussion}

Due to relatively weak exchange interaction of conducting holes with Mn impurities, samples under study can be considered as a conventional QW perturbed by magnetic layer. Thus, the comparison of our data with results for conventional QW with 2D hole gas should help us to distinguish phenomena related to the magnetic nature of $\mathrm{Mn}$ impurity.

One of the most common feature of diluted magnetic semiconductor systems is the appearance of local maximum (peak) on the temperature dependence of resistivity near $T_{c}$, which is related to substantial growth of critical fluctuation density in the vicinity of transition to longrange ordered state. Observation of this peak for the investigated samples at $T \approx 26 \mathrm{~K}$ (Fig. 1) indicates the presence of significant hole scattering on the magnetic subsystem. Actual value of $T_{c} \approx 20 \mathrm{~K}$ can be estimated from the maximum on the temperature dependence of $d \rho / d T$ [11]. The mismatch of $T_{c}$ and $\rho_{x x}(T)$ peak position is common for inhomogeneous systems.

One can see in Fig. 1 that below $T_{\text {min }} \approx 12.5 \mathrm{~K}$ the value of $\rho_{x x}$ begin to grow upon cooling, which is related to quantum corrections to conductivity. Comparing $\rho_{x x}(T)$ curves for $B=0$ and 3T, we can distinguish ranges of positive and negative magnetoresistance (MR), separated by $T_{M R}=31.5 \mathrm{~K}$.

It is important to note, that positive MR is quadratic, while low-temperature MR (Fig. 2a) is a superposition of negative MR in the whole range of magnetic fields and $\mathrm{SdH}$ oscillations that occur in high field region. Sharp MR in low field region is due to weak localization (WL). MR
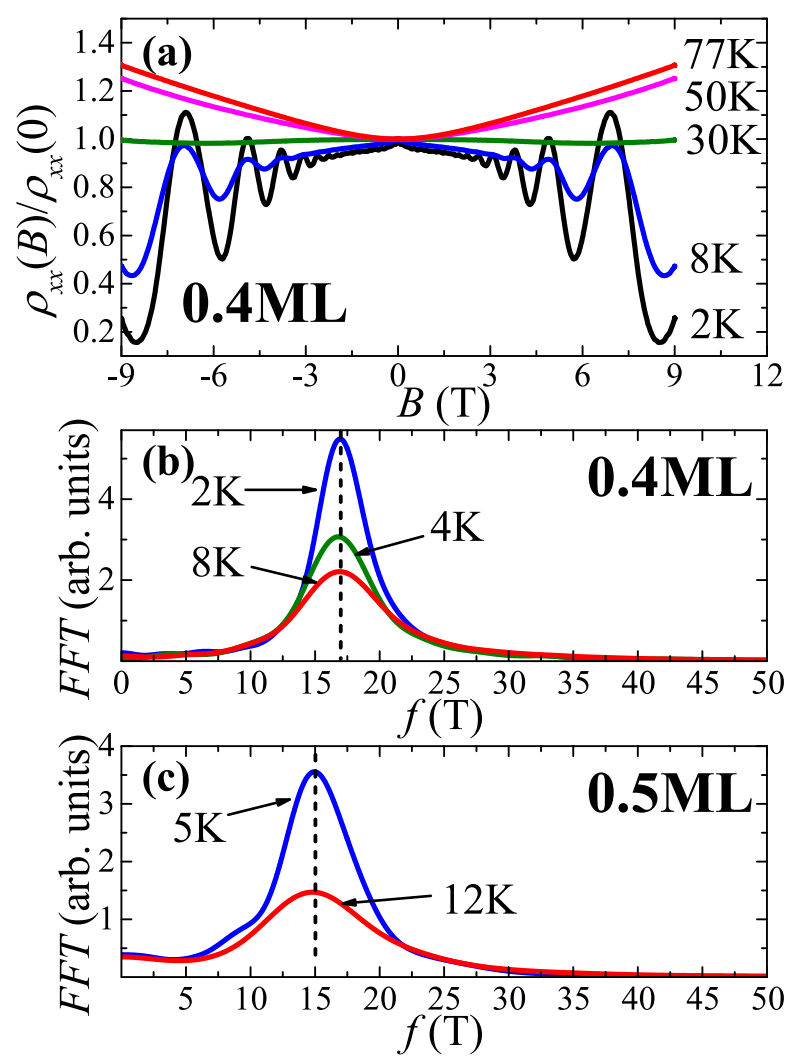

Figure 2. (colour online) (a) Magnetoresistance of 0.4ML sample at different temperatures. Fast Fourier Transform of SdH oscillation (after subtracting background MR) for 0.4ML (b) and $0.5 \mathrm{ML}$ (c) samples at different temperatures.

in the intermediate region and non-oscillating part of highfield MR have rather complicated functional form, which, however, cannot be described well by negative quadratic contribution. Thus, the spin-dependent scattering can be considered as the main reason of negative MR for $B>1 \mathrm{~T}$ at $T<30 \mathrm{~K}$. This assumption is in a good agreement with the aforementioned observation of peak in $\rho_{x x}(T)$. For similar non-magnetic structures negative quadratic MR was ascribed to diffusive $h$ - $h$ interaction correction [12].

Observed $\mathrm{SdH}$ oscillations were treated in the same way as in Ref's [13, 14]. SdH oscillation frequencies derived by Fast Fourier Transform (FFT) are temperatureindependent inside the experimental errors margins for both samples in the investigated temperature interval as it is shown in Fig. 2b and 2c. Assuming the hole spectrum of structures is spin-degenerate, we obtained carrier concentrations $p_{S d H}=0.82$ and $0.72 \cdot 10^{12} \mathrm{~cm}^{-2}$ for $0.4 \mathrm{ML}$ and $0.5 \mathrm{ML}$ samples, correspondingly. Comparing these with values obtained from normal Hall effect $p_{\text {Hall }}=0.96$ and $0.72 \cdot 10^{12} \mathrm{~cm}^{-2}(0.4 \mathrm{ML}$ and $0.5 \mathrm{ML})$ one can see, that for $0.5 \mathrm{ML}$ sample there is an excellent agreement, while $15 \%$ discrepancy for $0.4 \mathrm{ML}$ sample indicates the occupation of second subband, that didn't reveal itself in $\mathrm{SdH}$ oscillations. As it was said earlier, Hall mobilities $\mu_{\text {Hall }}$ $=2100$ and $1900 \mathrm{~cm}^{2} /(\mathrm{V} \cdot \mathrm{s})$ for $0.4 \mathrm{ML}$ and $0.5 \mathrm{ML}$ samples are significantly lower than values needed to fulfil $\mu B \geq 1$ condition for the observed $\mathrm{SdH}$ oscillations [10]. Thus, Hall effect includes average parameters of conduction channel, while $\mathrm{SdH}$ effect describes high-mobility regions. By exploring the temperature dependence of $\mathrm{SdH}$ 

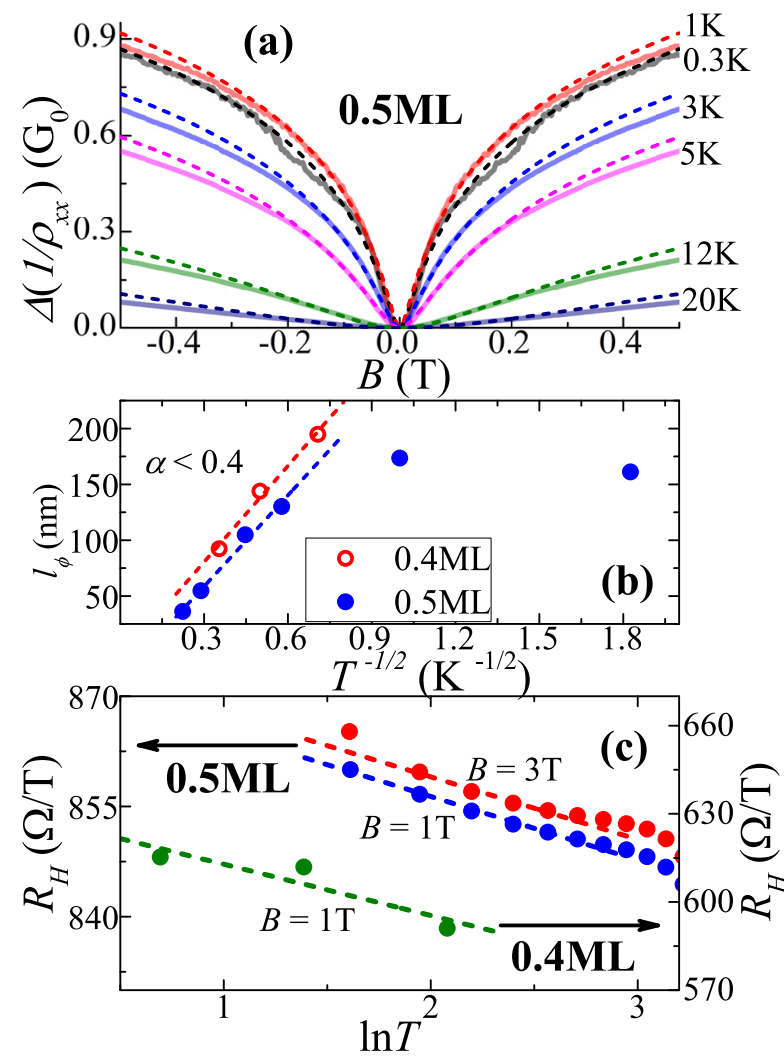

Figure 3. (colour online) (a) Inverse magnetoresistance of $0.5 \mathrm{ML}$ sample at different temperatures. Solid curves - experimental data, dashed lines - fitting by HLN formula (1). (b) Temperature dependence of the dephasing length $l_{\phi}$. Dashed lines linear fitting of $T>2 \mathrm{~K}$ range. (c) Temperature dependencies of Hall coefficient $R_{H}$ derived from different field ranges. Dashed lines - linear fit in low temperature region.

oscillation amplitudes, we calculated the effective mass of carriers $m^{*} \approx 0.15 m_{0}$ ( $m_{0}$ - free electron mass). This value is rather close to that reported for non-magnetic QW [12].

Low-field MR plotted in Fig. 3a has rather distinctive functional form for both samples, which is usually related to the damping of WL correction by magnetic field. It is important to note, that characteristic parameter of WL correction $B_{t r}=\hbar /\left(2 e l^{2}\right)$ (with $\hbar$ - Plank constant, $e$ - electron charge, $l$ - mean free path) for samples under study is approximately equal to $0.3 \mathrm{~T}$ and $0.45 \mathrm{~T}$ for $0.4 \mathrm{ML}$ and $0.5 \mathrm{ML}$ samples correspondingly for Hall parameters presented earlier. It should be mentioned, that for these samples we didn't observe any traces of weak antilocalization (WAL) effect, that was clearly seen for similar nonmagnetic structures with close parameters [15].

In order to investigate WL correction parameters in our samples we used common Hikami-Larkin-Nagaoki (HLN) formula [16] for 2D systems as a fitting function of lowfield MR. In diffusive regime corresponding MR can be described in the following way:

$$
\frac{\Delta\left(1 / \rho_{x x}(B)\right)}{G_{0}}=\alpha\left[\psi\left(\frac{1}{2}+\frac{\hbar}{4 e l_{\phi}^{2} B}\right)-\ln \left(\frac{\hbar}{4 e l_{\phi}^{2} B}\right)\right],
$$

where $\psi$ - digamma function, $G_{0}=e^{2} /\left(2 \pi^{2} \hbar\right), l_{\phi}$ - dephasing length, $\alpha$ - prefactor. One can see that fitting curves are in a good agreement with experimental data in the field range of $B \leq \pm 0.3 B_{t r}$ (Fig. 3a). Obtained $l_{\phi}(T)$ dependencies are plotted in Fig. 3b. It is clearly seen that $l_{\phi} \sim T^{-1 / 2}$ at $T>2 \mathrm{~K}$ for both samples. This behaviour usually indicates that inelastic $e-e(h-h)$ scattering is the main dephasing mechanism [17], which is common for 2D systems. But below $2 \mathrm{~K}$ the dependence $l_{\phi}(T)$ demonstrates saturation, which can be attributed both to conventional disorder and to Mn-related effects. However, the absence of this well pronounced saturation in nonmagnetic samples (see Fig. 4 in Ref. [15]), where $\tau_{\phi} \sim l_{\phi}^{2} \sim 1 / T$ behavior persists down to $0.4 \mathrm{~K}$ for all samples, favours the latter assumption. Another peculiarity of the observed WL is prefactor values. Theory predicts $\alpha=1$, as a mark that whole group of carriers contribute to WL $[17,18]$. Also there are several effects, that can be responsible for lower experimental $\alpha$ values [18]. But none of these effects can decisively explain $\alpha<0.4$ observed for our samples. Therefore, it can be related to the presence of Mn-layer. This assumption is partially supported by the absence of WAL effect.

It is commonly known that in $2 \mathrm{D}$ systems $e-e$ interaction (or $h$ - $h$ interaction in our case) can be significant and the relation between interference (WL and WAL) and interaction corrections should be derived from the experiment in every particular case [17]. The contribution of $h-h$ interaction in the diffusive channel is given by

$$
\delta \sigma^{h-h}=K_{h-h} G_{0} \ln \left(\frac{k_{B} T \tau}{\hbar}\right),
$$

where $k_{B}$ - Boltzman constant, $T$ - temperature, $\tau$ - transport relaxation time, and $K_{h-h}$ is the prefactor value, which is usually close to unity. A diffusive contribution should dominate in the interaction correction (favouring the drop of conductivity upon cooling) at $k_{B} T \tau / \hbar \ll 1$, which is for our samples is equal to $T \ll 45 \mathrm{~K}$, for given Hall parameters. There are three basic approaches to determine $K_{h-h}$ value from the experimental data. One of them is to investigate the logarithmical part of $\sigma_{x x}(T)$ dependence, assuming that $\sigma_{D}$ (Drude conductivity) in studied region is temperature independent and changes of $\sigma_{x x}$ are associated solely with the sum of interference and interaction correction. This approach is not applicable in our case because of the presence of a resistivity peak related to the critical fluctuations of magnetic subsystem. This peak can be very broad in inhomogeneous systems leading to additional temperature dependence of conductivity down to lowest temperatures. Indeed, using obtained parameters of WL we calculated the corresponding correction, taking it into account remaining part of conductivity changes derivative sign, i.e. below $T_{c}$ conductivity increases upon cooling without upturns of well pronounced saturation region. In frame of the second approach of $K_{h-h}$ determination one should examine negative quadratic MR related to $h$ - $h$ interaction. However, as it was said earlier, negative MR in our case is not quadratic and ascribed to spin dependent scattering. As far as we know the most suitable way to determine $K_{h-h}$ in our case is to examine $R_{H}(T)$ dependencies. The diffusive $h$ - $h$ interaction does not give any contribution to $\sigma_{x y}$. Using standard relations between conductivity and resistivity tensors one can see that in this 
case interaction correction can be observed in Hall coefficient $R_{H}$ [19]:

$$
\frac{\delta R_{H}}{R_{H}}=-2 \frac{\delta \sigma^{h-h}}{\sigma_{D}},
$$

Thus, in 2D system $R_{H}(T)$ dependence should demonstrate logarithmical increase upon cooling. In Fig. 3c corresponding dependencies are shown. One can see that for $0.5 \mathrm{ML}$ sample there is a difference of $R_{H}(T)$ derived by linear approximation of $\pm 1 \mathrm{~T}$ and $\pm 3 \mathrm{~T}$ field intervals of $R_{x y}$, which can be related to the presence of AHE contribution, while logarithmical slope of low-temperature region is the same for both curves. It should be noted that the positive value $R_{H}(3 T)-R_{H}(1 T)>0$ indicates the presence of negative AHE contribution, though to investigate it's actual dependence on $B$ and $T$ an additional data processing should be applied (see e.g. [2]). Logarithmic slope of presented dependencies was estimated in the same temperature range, for which $\mathrm{SdH}$ oscillations were investigated. Using the SdH frequency temperature independence we can assume that logarithmic change of $R_{H}(T)$ is related to $h$ - $h$ correction. Thus, applying relations (2) and (3) we have obtained prefactor values $K_{h-h} \approx 0.4$ and 0.1 for $0.4 \mathrm{ML}$ and $0.5 \mathrm{ML}$ samples, correspondingly. The value of $K_{h-h} \approx 0.2-0.3$ was reported for similar non-magnetic structures in Ref. [12], if one uses the relation between $K_{h-h}$ and Fermi liquid parameter $F_{0}^{\sigma}$. Although $R_{H}$ data presented in that work wasn't discussed one can easily see that applying the same relations (2) and (3) yields sufficiently larger $K_{h-h}$ value (by a factor of 2 under our estimations). Thereby the exact impact of Mn layer on $h-h$ interaction in QW cannot be formulated from the current data and needs further investigation. The discrepancy of $K_{h-h}$ for studied samples can be attributed to the occupation of second subband in $0.4 \mathrm{ML}$ sample and presumably different states of magnetic subsystems at different Mn concentrations.

Finally, we can compare different length scales observed for samples under study. For simplicity we will use data for $0.5 \mathrm{ML}$ sample. Magnetic length corresponding to $\mathrm{SdH}$ oscillations onset at $5 \mathrm{~K}$ is about $17 \mathrm{~nm}$ [10], which is rough estimation for minimum size of high mobility regions (metallic droplet in QW). Even for Hall parameters mean free path is about $26 \mathrm{~nm}$, while for parameters of high-conductive droplet it can be even larger. Thus, at zero field these droplets can be considered as areas of "ballistic" transport, which can affect different properties of system, e.g. interaction correction. For example, the logarithmic growth of $R_{H}(T)$ starts to be significant below $12 \mathrm{~K}$, while corresponding $L_{T}=\left(D \hbar / k_{B} T\right)^{1 / 2}(D$ - diffusion coefficient) at $12 \mathrm{~K}$ is equal to $52 \mathrm{~nm}$. Thus, rather low temperature of interaction correction onset and low values of $K_{h-h}$ can be related to the contribution of these ballistic areas (for which $k_{B} T \tau / \hbar>1$ ) that have an opposite sigh compared to the diffusive one. Also, if we assume that saturation of $l_{\phi}(T)$ dependence is related to the spin-dependent scattering becoming main dephasing mechanism, then saturation value of $l_{\phi} \approx 170 \mathrm{~nm}$ gives us estimation of low-field correlation length of magnetic subsystem.

\section{Conclusions}

We have studied magnetoresistance and Hall effect of $\mathrm{GaAs} / \mathrm{In}_{x} \mathrm{Ga}_{1-x} \mathrm{As} \mathrm{QWs}$ with remote Mn impurity. The presence of resistivity peak in $\rho_{x x}(T)$ and negative MR for $B>1 \mathrm{~T}$ below $30 \mathrm{~K}$ indicates the significant spin-dependent scattering of conducting holes on magnetic subsystem, which is partially supported by the observed AHE contribution. Observation of $\mathrm{SdH}$ oscillations indicates the presence of several types of regions in conduction channel with significantly different hole mobilities. The absence of pronounced WAL effect along with abnormally low prefactors for WL and $h$ - $h$ interaction contribution can be attributed to the damping of quantum corrections by magnetic impurities. The saturation of the dephasing length upon cooling can be also related to the presence of $\mathrm{Mn}$ atoms. Although for determination of the exact impact of Mn impurities on quantum corrections to conductivity in studied systems further investigations are needed.

This work was partially supported by Russian Foundation for Basic Research (grant \#16-32-00446).

\section{References}

[1] A.M. Nazmul et. al., Phys. Rev. Lett. 95, 017201 (2005)

[2] L.N. Oveshnikov et. al., Sci. Rep. 5, 17158 (2015)

[3] L.N. Oveshnikov et al., JETP Lett. 100, 570 (2014)

[4] V.A. Kulbachinskii et.al., Low Temp. Phys. 33, 174 (2007)

[5] D. Neumaier et. al., Phys. Rev. Lett. 99, 116803 (2007)

[6] V.K. Dugaev et. al., Phys. Rev. Lett. 101, 129701 (2008)

[7] D. Neumaier et. al., Phys. Rev. Lett. 101, 129702 (2008)

[8] B.A. Aronzon et al., J. Appl. Phys. 107, 023905 (2010)

[9] V. Tripathi et al., Phys. Rev. B 84, 075305 (2011)

[10] L.N.Oveshnikov et al., Bull. Lebedev Phys. Inst. 44, 143 (2017)

[11] M. Wang et al., App. Phys. Lett. 104, 132406 (2014)

[12] G.M. Minkov et al., Phys. Rev. B 72, 165325 (2005)

[13] V.A. Kulbachinskii et al., Semiconductors 49, 921 (2015)

[14] V.A. Kulbachinskii et al., Semiconductors 49, 199 (2015)

[15] G.M. Minkov et al., Phys. Rev. B 71, 165312 (2005)

[16] S. Hikami et al., Prog. Theor. Phys. 63, 707 (1980)

[17] B.L. Altshuler, A.G. Aronov, Electron-electron interaction in disordered conductors (Elsevier, Amsterdam, 1985)

[18] G.M. Minkov et al., Phys. Rev. B 70, 245423 (2004)

[19] A.Yu. Kuntsevich et al., Phys. Rev. B 94, 235401 (2016) 